\title{
tic\&société
}

Vol. $4, n^{\circ} 1 \mid 2010$

Interactivité et lien social

\section{Formes de réception et d'appropriation des ressources numériques en milieu étudiant.}

Enquête ethnographique en bibliothèque universitaire

\section{Mariangela Roselli}

\section{OpenEdition}

\section{Journals}

Édition électronique

URL : http://journals.openedition.org/ticetsociete/824

DOI : 10.4000/ticetsociete.824

\section{Éditeur}

Association ARTIC

Référence électronique

Mariangela Roselli, «Formes de réception et d'appropriation des ressources numériques en milieu étudiant. », tic\&société [En ligne], Vol. 4, n 1 | 2010, mis en ligne le 19 mai 2010, consulté le 19 avril 2019. URL : http://journals.openedition.org/ticetsociete/824 ; DOI : 10.4000/ticetsociete.824 
tic\&société - 4 (1), 2010

\title{
Formes de réception et d'appropriation des ressources numériques en milieu étudiant
}

Enquête ethnographique en bibliothèque universitaire

\author{
Mariangela Roselli \\ Enseignante-chercheure en sociologie \\ Université de Toulouse2-Le Mirail \\ CERTOP UMR CNRS 5044 \\ 5, allées Antonio Machado \\ 31058 - Toulouse cedex 09 \\ FRANCE \\ roselli@univ-tlse2.fr
}




\section{Formes de réception et d'appropriation des ressources numériques en milieu étudiant} Enquête ethnographique en bibliothèque universitaire

Résumé : Une enquête ethnographique sur les publics de la bibliothèque de l'Université de Toulouse-le Mirail (lettres et sciences humaines) réalisée en 2007 et 2008 a permis d'étudier les formes de réception de l'environnement numérisé par les étudiants. Deux scènes observées et deux portraits sociologiques d'usagers sont proposés à titre illustratif des nouvelles pratiques en bibliothèque. Les cas qui ont été choisis montrent l'émergence de rationalités de recherche liées à l'environnement numérique et la nécessité d'une propédeutique des recherches documentaires en ligne.

Mots-clés: étudiants, jeunes chercheurs, nouvelles pratiques, environnement numérique, bibliothèques universitaires, nouveaux usagers.

Abstract : An ethnographical inquiry on the users of the university library of the University of Toulouse (France, literature and human sciences) carried out in 2007-2008 shows how students do with the Web environment and electronical resources. Two ethnographical scenes and two sociological portraits are presented to illustrate how computers and numerical resources have generated new practices in university library. The cases described in the paper develop some examples of the new rationalities in scientific research associated with the new environment. They clearly show that a foundamental teaching in numerical environment and research methodology is necessary.

Key words : students, young researchers, new practices, numerical environment, university libraries, new users.

Resumen: Un estudio etnográfico sobre la Biblioteca Pública de la Universidad de Toulouse le Mirail (Letras y Ciencias Humanas) realizado en 2.007 y 2.008 permite estudiar la manera en la que los estudiantes perciben, reciben y usan los recursos electrónicos. Se observan dos escenas y dos retratos sociológicos de los usuarios propuestos como ejemplo (la traducción exacta es a título ilustrativo pero en español se dice «como ejemplo «) de las nuevas prácticas en la biblioteca universitaria. Los casos que se eligieron 
Mariangela ROSELLI

muestran la emergencia lógica de la organización del tiempo y de las actividades en Internet unido al entorno informático. Se plantean también la cuestión de la necesidad de una formación para la investigación documental en línea.

Palabras esenciales : estudiantes, jóvenes investigadores, nuevos usos, entorno digital bibliotecas universitarias y nuevos usuarios. 
Formes de réception et d'appropriation des ressources numériques en milieu étudiant

Les récentes enquêtes sur les publics des bibliothèques universitaires (BU) en France montrent que celles-ci sont fréquentées par des publics très divers qui développent des pratiques des lieux, des collections et des services très hétérogènes (Petite et Le Douarin, 2008 ; Enquête Paris 8, 2007 ; Maresca, 2005). Les enquêtes sur les pratiques internautiques en bibliothèque (Ghitalla et al., 2003) indiquent par ailleurs une activité de lecture à la fois persistante et multimodale. Héritière de conventions et de rhétoriques historiquement ancrées (Chartier, 1996), l'activité à laquelle se livrent lecteurs et internautes combine acquis traditionnels du rapport à l'écrit papier et innovations audiovisuelles et manipulatoires. C'est ce que remarquent les auteurs des travaux portant plus particulièrement sur les bibliothèques universitaires (Brodbent et Cara, 2003 ; Maresca, 2005), à savoir la permanence de modèles traditionnels de la lectureétude (sélective, synoptique, analytique) et le caractère hybride de la recherche documentaire, héritière des index et des catalogues papier mais déjà ouverte à une logique de navigation.

Dans le cadre d'un contrat de recherche soumis à appel d'offres par le Service commun de la documentation de l'Université de Toulouse-le Mirail, nous avons mené une enquête sur les publics et les usages de la Bibliothèque Universitaire (Roselli et Perrenoud, 2008). Partis de l'observation des pratiques en situation, nous en sommes venus à faire une sociologie des usages attentive aux multiples processus d'intermédiation entre un usager-acteur (centre d'intérêt, projet d'usages, négociation) et l'outil (Jouët, 2000). L'inscription dans une sociologie des usages s'est faite autour de la notion de l'usager, pratiquant actif, qui s'approprie les outils à des fins d'émancipation personnelle. Dans le domaine de la lecture, M. de Certeau (1980) a depuis longtemps montré l'intérêt des procédures de contournement des usages prescrits, des formes d'inventivité des pratiques ordinaires de lecture. Si ce dernier a essentiellement traité de l'activité de lecture, du braconnage qui s'y opère à travers des procédures d'appropriation du texte, l'intérêt qu'il porte "aux manières de faire ", aux gestes a priori insignifiants et aux tactiques mises en œuvre par les usagers comme autant de formes de microrésistances à l'imposition de normes, s'avère aussi pertinent pour les supports traditionnels que pour les médias informatisés. La notion d'usage a d'ailleurs contribué à éclairer les conditions d'insertion des technologies de l'information dans les pratiques ordinaires.

Les enquêtes sur le public à l'intérieur desquelles se situe cette recherche s'inscrivent dans une tradition sociologique qui, depuis l'enquête sur les images et les bibliothèques de J.-C. Passeron et M. Grumbach (1984), explique l'hétérogénéité des publics par la singularité de la relation à l'œuvre et par l'intention subjective associée aux pratiques culturelles. Même en présence 
Mariangela ROSELLI

d'une technicité très élevée comme celle des médias informatisés, la pratique effective et subjective ne peut être formatée, relevant de l'appropriation et de la contextualisation des usages dans le corps de pratiques sociales et individuelles. Tout comme le lecteur qui construit par l'interprétation une relation singulière au texte, l'usager négocie son activité entre son projet et le dispositif (Souchier et al., 2003) : la manière dont il s'approprie ce qu'il voit et lit doit être étudiée à partir des raisons qui le poussent à s'engager dans une activité, laquelle prend forme dans un cadre social précis. Dans cette optique, se dessine une lecture moins centrée sur un texte restreint, stabilisé et signé, et un lecteur qui construit lui-même son texte à partir d'un but, d'un centre d'intérêt et d'une stratégie de détection des documents. En cela, nous retrouvons l'hypothèse d'un récepteur fort, selon le mot de J.-C. Passeron, pour qui les degrés d'expertise se lisent moins dans la manière de "consommer " un objet culturel que de le «produire » en l'adaptant à des possibilités réalistes d'interprétation (Passeron, 1991).

S'inscrivant à la fois dans une sociologie de la réception et des usages, l'enquête en BU s'est concentrée sur les pratiques effectives et nous a introduits aux différentes facettes du processus d'appropriation, dans la double dimension cognitive (apprentissage de connaissances, acculturation à un savoir-faire spécifique et incorporation de gestes nouveaux) et empirique (gestes et postures corporelles, maniement des supports, situations individuelles et collectives). Elle a permis de comprendre l'importance prise par les cheminements de recherche individuels avec les technologies de l'information et de la communication (TIC) : fonctionnant comme des méthodes de travail, ces cheminements donnent à voir des modes de lecture plus composites, qui combinent l'audiovisuel et le lien hypertexte avec l'impression de documents et la lecture sur imprimé. Les modalités de lecture qui en résultent exigent de l'usager - même du moins expert - d'être personnellement engagé dans la construction des documents à partir desquels il travaille. Cet engagement, investissement tout à la fois intellectuel et affectif, distinguerait les pratiques des uns et des autres ; il agirait comme un mécanisme différenciateur des logiques d'usage et des enjeux que les individus investissent dans le temps consacré à la lecture-étude.

\section{Méthodologie d'enquête et contexte}

Après avoir décrit les présupposés théoriques qui ont guidé la mise en place de l'enquête, nous présentons le contexte et la démarche méthodologique. Nous montrons en particulier l'intérêt de la posture ethnographique pour comprendre la spécificité des pratiques internautiques en BU. 
Formes de réception et d'appropriation des ressources numériques en milieu étudiant

\subsection{Protocole d'e`quête et posture eth`ographique}

Afin de mieux appréhender les publics hétérodoxes qui peuplent les bibliothèques universitaires, nous avons cherché à nous démarquer de l'approche classique par les statistiques de fréquentation en élaborant un protocole ethnographique. Celui-ci est caractérisé par plusieurs exigences : une présence longue sur le terrain (18 mois); deux phases d'observations, une statique, l'autre dynamique; l'entretien semi-directif pour mieux connaître l'usager observé; le croisement des données. Cette démarche s'avère particulièrement féconde lorsqu'elle utilise de manière complémentaire des données statistiques et les résultats issus des autres recherches. C'est ce que propose le texte proposé ici.

Dans un premier temps, nous avons consacré six mois à l'observation statique des étages, espaces, couloirs, escaliers, hall d'entrée, salle d'étude annexe et autres zones de la BU. Entre janvier et juin 2007, nous nous sommes rendus à la $\mathrm{BU}$ deux à trois jours par semaine, trois à six heures par jour pour un total d'environ 170 heures d'observation. La plupart du temps, l'observation était menée depuis un poste de travail parmi les usagers (table dans les salles de lecture, poste informatique) en situation masquée. Les deux enquêteurs en situation d'observation ont commencé par tenir un journal de terrain ; celui-ci a permis d'élaborer une grille d'observation articulée autour de trois catégories d'indicateurs : les supports, les modes de travail, les activités. La question qui a guidé cette phase concerne les gestes et les activités associés à des espaces et à des dispositifs spécifiques (écrans, ressources électroniques, collections imprimées). À partir de ce matériau déjà très riche, des styles d'usage ont commencé à se faire jour en relation avec les lieux permettant de dégager une géographie humaine de la BU.

Après la phase d'observation des lieux, la phase dynamique de l'enquête a consisté à suivre, observer et interroger certains usagers au cours d'une de leur visite à la bibliothèque. Malgré l'impression de flux continu d'étudiants, une BU a "son » public régulier. Les mois passés en observation ont servi à repérer les usagers assidus, soit qu'ils séjournent soit qu'ils empruntent, selon la distinction établie par Passeron et Grumbach. La connaissance des lieux et de leurs usages a ainsi permis de cibler les observations autour de certains usagers que nous avons suivis. Nous avons appelé cette méthode, tracking ethnographique. L'idée du tracking est venue des premières observations qui ont fait ressortir l'importance des "parcours" des usagers, entendus à la fois comme déplacements dans l'espace physique de la bibliothèque et comme cheminements de recherche entrepris pour détecter des documents (papier et électroniques). Suivant la méthode de l'eye tracking expérimentée dans les 
Mariangela ROSELLI

enquêtes sur le Web en station publique (Ghitalla et al., 2003) et la méthode ethnographique plus classique qui consiste à suivre à la trace les individus, nous avons élaboré la méthode du tracking ethnographique d'utilisateurs en prise avec les dispositifs proposés en bibliothèque.

Le choix des personnes à interroger s'est fait selon deux critères : étant dans une perspective qualitative, nous avons privilégié la diversité et la singularité. Dans cette optique, la population à enquêter tend à couvrir la plus vaste étendue possible au départ et elle se restreint au fur et à mesure que l'on rencontre de plus en plus de cas similaires. En entretien, nous avons choisi de partir de ce que les usagers font et de leur parole, en nous plaçant à proximité de leurs objets de travail. Nous avons essayé de créer une situation de continuité avec les pratiques immédiatement observées pour que la personne ne puisse plus être dans le seul discours car ses objets, ses manipulations, ses mouvements risquent de la contredire. Pour les usagers des postes informatiques, par exemple, l'entretien a commencé par un retour commenté sur la démarche de recherche effectuée, avec description de la demande initiale, des moteurs de recherche ou portails utilisés, des niveaux d'écran ouverts, du jeu de renvois et de l'arborescence suivie. Nous avons prêté une attention particulière à la qualification spontanée par les enquêtés de leurs habitudes, de leurs manières d'étudier, de leur carrière scolaire, de leurs modes de sociabilité à l'université et en dehors. Ce faisant nous avons pu saisir l'usager de la BU non seulement dans les murs mais hors des murs, sur le campus, à la ville, au domicile afin de comprendre la place occupée dans son quotidien par la bibliothèque, les livres et Internet. C'est à partir de ce matériau que nous avons pu dresser une soixantaine de portraits sociologiques selon la méthode utilisée par B. Lahire dans Tableaux de familles (1995). Le portrait sociologique est une méthode qui restitue l'imbrication unique des contextes sociaux chez un même individu. Ce style d'écriture, proche de l'essai, tente de situer l'individu dans l'action pour donner à voir les effets de la socialisation antérieure.

\subsection{Le co' texte de l'e' quête}

L'Université de Toulouse 2-Le Mirail est une grande université publique de lettres et de sciences humaines qui compte plus de 20000 étudiants. Ce campus reçoit chaque année des milliers de lycéens qui sont, dès le premier semestre, confrontés à des enseignements ex-cathedra (cours magistraux et travaux dirigés également) très riches en contenus et en références bibliographiques. Laissant les étudiants libres de s'organiser dans le travail d'étude, ce type d'enseignement présuppose une discipline intellectuelle et méthodologique que tous les bacheliers n'ont pas. C'est donc aux étudiants eux-mêmes de développer une capacité à s'organiser après et autour des

tic\&société - 4 (1), 2010 
Formes de réception et d'appropriation des ressources numériques en milieu étudiant

cours, de manière à être rapidement autonomes dans le travail d'étude. C'est précisément au cours du premier "semestre " (septembre à décembre) que cette autonomie doit se mettre en place pour que les étapes du cursus puissent se succéder sans écueil : dans la réussite universitaire, le facteur de prise en charge personnelle entre en jeu dès le premier mois : alors que les bacheliers s'adaptent au système et cherchent leurs marques, cette capacité à être autonome dans le travail personnel détermine souvent la suite de la carrière d'étudiant.

Des disciplines aussi singulières que la psychanalyse, la musicologie, le cinéma, l'histoire de l'art, les arts plastiques y côtoient les langues étrangères, le FLE (Français Langue Étrangère), les lettres (anciennes et modernes), l'histoire, la géographie, la sociologie, les sciences économiques, les statistiques appliquées aux sciences humaines. Située à l'entrée de l'université, la nouvelle BU a ouvert ses portes en novembre 2004. C'est dans ce bâtiment, moderne et aérien, que s'est déroulée l'enquête : quatre étages accessibles au public, des kilomètres linéaires de collections imprimées qui poussent les usagers progressivement contre les baies vitrées et les murs, des postes informatiques et des postes de connexion littéralement pris d'assaut.

Une bibliothèque n'est pas un espace neutre; dans son organisation matérielle et spatiale, elle exprime tout autant une vision de l'organisation du savoir et une conception de la relation au public (Le Marec et Babou, 2003). Cette organisation fonctionne comme cadre sémiotique au sens où il fournit des indices symbolisant l'ordre selon lequel sont rangés les objets ainsi que le sens émanant de cet ordre (imprimés omniprésents au détriment des ressources électroniques; place allouée aux collections plus importante que celle laissée aux usagers). Cette mise en ordre du savoir dégage une intention normative et prescriptive que les usagers peuvent tordre, en tant que lecteurs créatifs et non seulement coopératifs. Dans un cas comme dans l'autre (conformité aux attentes ou appropriation par usages détournés), ce cadre est symbolique et est évidemment en lien étroit avec les manières d'être et de faire en bibliothèque que nous avons pu analyser.

\section{L'Internet à la BU, l'information d'abord}

L'avènement depuis une dizaine d'années d'un autre type de supports, informatique d'abord, numérique ensuite, et l'émergence, surtout chez les jeunes générations, de nouvelles habitudes de travail sur écran, ne permettent plus d'étudier les compétences des jeunes étudiants en lettres uniquement en termes de lecture des textes d'auteurs. Certes, dans le contexte particulier des études, la prédominance de l'écrit sur feuille, l'habitude et la consigne du travail 
Mariangela ROSELLI

sur manuel, les modalités d'évaluation individuelles et manuscrites, obligent les lycéens devenus étudiants à pratiquer constamment des transferts de savoir et de savoir-faire pour s'adapter aux exigences institutionnelles. II reste toutefois que des profils hybrides apparaissent, à l'aise avec l'Internet et négociant des modalités d'appropriation des textes selon des formes consacrées (citations, extraits commentés, renvois en notes, références bibliographiques et cumulation des connaissances). Ces figures "mixtes» mi-lecteur, mi-internaute se retrouvent tout particulièrement en $\mathrm{BU}$ où une certaine liberté d'accès, de séjour et de déambulation permet d'occuper une place d'usager tout en faisant bouger les frontières des usages. En cela, la qualification de "bibliothèque hybride » en référence à l'environnement numérisé répond déjà à une réalité des usagers.

Cette partie montre que, si l'environnement numérisé a contribué à exporter l'offre de lecture hors des murs de la bibliothèque, l'espace physique de la bibliothèque a gagné un plus large public grâce à l'accès à Internet. Pour ce public, la tendance "cybercafé » n'est pas seulement un détournement des services de la BU, mais une façon d'intégrer à l'univers savant une dimension plus conviviale et plus vivante : interactions, groupes, déambulations cohabitent avec des postures de lecture plus traditionnelles (silence, solitude, concentration). Dans le même temps, ce public d'internautes, de plus en plus nombreux dans l'espace appelé "Recherche documentaire informatisée », n'a rien en commun avec les usagers de l'offre numérique documentaire qui, eux, ont plutôt tendance à déserter le lieu en consultant à distance l'environnement numérisé de la BU.

\subsection{La BU re' due plus viva' te grâce à I'l' ter' et}

Une qualification de la bibliothèque récurrente dans les entretiens recueillis au cours de l'enquête a opposé la bibliothèque "temple " d'autrefois à la bibliothèque "lieu vivant " d'aujourd'hui. Pour un grand nombre des usagers interrogés, les écrans sont le principal support utilisé en BU et l'Internet leur première raison de la fréquenter. En clair, l'Internet importe dans les murs des bibliothèques des flux de visiteurs et des façons de faire auxquelles les bibliothécaires ne sont pas toujours préparés ; ces usages entrent parfois en dissonance avec l'organisation des lieux (Desprès-Lonnet, 2007). Malgré l'effet de filtre qui joue en amont, l'ouverture des postes informatiques a produit deux phénomènes inédits: la fréquentation de certains espaces de la $\mathrm{BU}$ par des personnes extérieures au monde étudiant et au monde lettré en général et la masculinisation du public qui séjourne longtemps en bibliothèque (Maresca et al., 2007). Ces deux phénomènes se matérialisent sous différentes formes dans les espaces physiques. 
Formes de réception et d'appropriation des ressources numériques en milieu étudiant

Au cours des 18 mois passés à enquêter, nous avons remarqué la récurrence de trois phénomènes : premièrement, le nombre élevé d'utilisateurs informatiques, deuxièmement, le "détournement» - selon le mot des bibliothécaires - des services informatiques destinés aux consultations documentaires au profit d'utilisations mixtes et, troisièmement, les frontières territoriales qui tendent à s'installer entre différents types de publics. Ces trois phénomènes, s'ils ont été étudiés en médiathèque (Maresca et al., 2007), ont peu été évoqués en $\mathrm{BU}$. Or il semble bien que la consultation internautique à la $\mathrm{BU}$, telle qu'elle est développée par les jeunes (étudiants), présente des traits spécifiques. L'une de ses principales caractéristiques est l'articulation étroite entre dimension ludique et dimension savante, temps intime et temps public, pratique privée et pratique studieuse. A partir de deux scènes observées, nous allons montrer comment se construit ce continuum entre sphères d'intérêts et domaines de compétences. Celles-ci, loin d'être exclusivement liées aux loisirs culturels (musique, images, espaces virtuels, jeux), servent également aux étudiants pour aborder d'une façon particulière les connaissances et effectuer le travail d'étude : les compétences développées sur la toile à l'occasion de ces pratiques constituent des rationalisations, des adaptations d'un environnement complexe, voire inconnu pour certains, et peuvent permettre une insertion progressive dans le travail documentaire "sérieux ». Le second trait saillant et spécifique concerne la géographie ségrégative des utilisateurs (Poissenot et Ranjard, 2005) : selon que l'on est plutôt dans une utilisation documentaire ou informationnelle de la bibliothèque, les espaces fréquentés et les manières de faire ne se ressemblent pas. On peut même affirmer que ces deux types d'usager vivent sur deux territoires fermés, qui se côtoient sans se voir.

Cette population, à la fois plus importante et nouvelle dans ses habitudes et comportements, a grandement modifié l'ambiance, aux dires des bibliothécaires et des usagers eux-mêmes. Ces derniers se sentent moins contrôlés, moins astreints à lire des ouvrages : avec l'Internet mis à disposition, les postures audiovisuelles sur écran sont admises, dans les variantes individuelles et collectives. Surtout, ce que l'écran semble avoir ajouté est une mince cloison d'intimité qui permet d'éviter le regard frontal entre usagers eux-mêmes et usager et bibliothécaire. II y a aussi ce que permet la navigation sur la toile en termes de débordement de l'usage documentaire et scientifique, de mélange des genres et des pratiques culturelles. Tout se passe comme si le poste informatique facilitait autant le séjour en BU que le travail d'étude : l'effort requis pour rester assis, concentré devant une tâche semble coûter moins lorsque les contenus et les supports passent par l'écran. Au lieu de dévier les étudiants des pratiques studieuses, les activités ludiques (culturelles ou relationnelles) sur Internet le détournent momentanément, lui apportent une légèreté qui l'aide à tenir. Mais que cherchent-ils en faisant des recherches? Sacrifient-ils leur 
Mariangela ROSELLI

temps et leur attention aux mondes virtuels, aux jeux, aux réseaux sociaux type MySpace-, aux PDA - type iPod -(Eisenberg, 2008) ? Ou alors surfent-ils dans une abondance informationnelle et communicationnelle (Lahary, 2008) qui permet de glisser, sans s'en apercevoir, d'un champ à un autre, d'un registre à un autre, d'une activité à une autre ?

Dans leur enquête de 2001 sur les usages d'Internet en bibliothèque (médiathèque et $\mathrm{BU}$ ), $\mathrm{E}$. Pedler et $\mathrm{O}$. Zerbib montrent que, contrairement aux autres fractions d'usagers des bibliothèques de l'enquête (adultes utilisant un écran en station publique), les étudiants ne se contentent pas de jouer sporadiquement aux jeux vidéo, puisqu'ils se montrent très nombreux à déclarer en faire un usage majoritaire à domicile : $44 \%$ des étudiants utilisent ainsi leur ordinateur domestique principalement à des fins ludiques, contre seulement $19 \%$ des actifs et assimilés. On sait que le marché des jeux vidéo constitue l'un des secteurs les plus en pointe de l'informatique puisqu'il engendre de très nombreuses innovations dans les modes de programmation. En privilégiant fortement les logiciels qui émanent de ce secteur, les étudiants accordent, comme dans le domaine des séries télévisées, une attention toute particulière aux productions ludiques les plus récentes de l'offre multimédia. En médiathèque et au domicile, les étudiants entraînent leur ordinateur dans une navigation plus aléatoire ; en $\mathrm{BU}$, en revanche, l'habitus lettré, le (risque de) contrôle et la posture studieuse comme modèle de référence contribuent à verrouiller relativement les pratiques sur Internet ; en tout cas, à faire apparaître dans cet espace difficile à privatiser seulement les utilisations les plus présentables et les plus dignes.

Place de travail, box privatisé, outil de recherche ou d'écriture, l'ordinateur change les configurations classiques de la bibliothèque universitaire donnant à voir, souvent, autant d'étudiants que d'étudiantes, assis en groupe, discutant à mi-voix sous l'œil inquiet des bibliothécaires. Les usagers des postes informatiques sont particulièrement intéressants pour une sociologie de la bibliothèque parce qu'ils incarnent, en forçant le trait, d'autres figures de lecteurs traditionnellement mal tolérées dans ces espaces savants. Ces profils d'usagers, marginaux par rapport au monde lettré, poussent les limites de la BU vers des utilisations détournées (Touitou, 2008) et forcent les bibliothécaires à accepter tout ce qui historiquement a été poussé vers la périphérie de la bibliothèque. En cela, ils constituent des publics à la fois nouveaux et directement produits par l'offre informatisée (Roselli, 2006). 
Formes de réception et d'appropriation des ressources numériques en milieu étudiant

Aux postes informatiques du service " Recherche documentaire informatisée »

\section{Mardi 17 avril 2007.}

A $10 \mathrm{~h} 30$, tous les postes informatiques en état de marche sont occupés, comme c'est le cas la plupart du temps entre $10 \mathrm{~h}$ et $16 \mathrm{~h}$. Tous les usagers sont seuls, pour l'instant. L'utilisation de ce service est massive et constante ; on observe aussi que la parité entre garçons et filles est atteinte au début de la séance d'observation. Comme dans d'autres espaces de la $\mathrm{BU}$, on peut s'interroger sur la topographie "sexuée " des lieux où les hommes sont parfois aussi nombreux que les femmes alors que sur l'ensemble de l'UTM on trouve presque trois étudiantes pour un étudiant et à la BU cinq étudiantes pour un seul étudiant. Ici, les utilisateurs des postes informatiques sont souvent des hommes, et un rapide tour des deux "îlots » de PC permet d'observer une très large consultation de sites en arabe. Dans l'aile sud du même étage, sur les quelques machines en libre accès pour le traitement de texte, bien protégées par des cloisons hautes, on trouve aussi des jeunes adultes naviguant sur Internet à des fins largement privées (recherche de billets d'avion low cost, locations de vacances, covoiturage pour des destinations éloignées, consultations des sites des agences de travail intérimaire). Au cours des deux heures d'observation, les interactions entre usagers et bibliothécaires ont été rares, la plupart d'entre elles étant des formes de contrôle (le site consulté ne semble pas être un site de recherche) ou de censure (trop de temps passé sur la même machine) ou encore des rappels au silence pour les groupes de deux ou trois personnes assises devant le même écran.

Sur les postes informatiques qui donnent un libre accès à Internet, on trouve, comme la scène observée permet de le voir, de jeunes adultes habitant dans le quartier du Mirail, (banlieue populaire de Toulouse, habitée par un grand nombre de familles issues de l'immigration), séparé de l'université par une passerelle piétonne. Ces jeunes adultes dérangent pour plusieurs raisons. La première est une raison de place. D'abord, parce qu'ils se comportent comme des "étrangers » vis-à-vis du monde universitaire, alors qu'ils sont parfois inscrits à la faculté. Par leurs attitudes éloignées des comportements attendus et leur apparence physique (origine, gestuelle, vêtements), ils incarnent une dissonance culturelle qui pousse les bibliothécaires à la vérification administrative (carte d'étudiant, carte de lecteur). Ce contrôle exacerbe les interactions ordinaires et, plus généralement, les rapports sociaux. Ensuite, se cantonnant à des utilisations privées et ludiques d'Internet, ils usent de l'accès 
Mariangela ROSELLI

libre aux outils multimédias, ce qui motive les bibliothécaires à intervenir pour réguler l'utilisation des postes, contrôler la nature des opérations, voire vérifier l'identité de l'usager. Autrement dit, ils provoquent le contrôle et la réprobation systématiques, qu'ils perçoivent comme une réponse de l'institution aux comportements populaires en bibliothèque, jugés alors comme inadéquats: gesticulations, sonorités, échanges, pratiques récréatives, accompagnent d'autant plus facilement les pratiques de recherche que celles-ci s'éloignent du modèle lettré (Chartier, 1996). La normalisation des façons de lire, processus historique qui continue de marquer les espaces physiques des bibliothèques, conduit logiquement les professionnels à percevoir certains comportements comme déviants vis-à-vis des normes en vigueur. Entre autres, l'usager doit donner à voir son rapport au travail par son hexis corporelle, dans le repli sur soi.

Les pratiques groupales et récréatives qui sont souvent associées à l'utilisation des postes informatiques rappellent dans une certaine mesure l'attitude populaire de réception de l'art et de la culture avant leur normalisation sous des formes cloisonnées et privées (musées, concerts). Tout se passe comme si la BU avait été victime d'un transfert imparfait (Lahire, 2004) : lieu de culture, on lui attribue une mission civique (d'information, d'intégration, d'insertion) parce qu'on y trouve l'Internet en libre accès (gratuit et public) et à proximité (la bibliothèque dans la cité). Les bibliothécaires se sentent dépassés par ce public qui rechigne à solliciter le personnel en s'affichant comme " indépendant ». La scène présentée plus haut montre que, autour des postes informatiques, les relations avec les bibliothécaires sont rares et ne concernent que rarement des demandes documentaires. Mais le plus difficile à accepter pour les bibliothécaires est le déni répété et revendiqué de la relation d'aide : alors qu'ils leur offrent des services de qualité, gratuits et fiables (au sens où ils sont mis à la disposition un grand nombre d'heures) et qu'ils se mettent euxmêmes à la disposition des usagers les moins préparés, éduqués ou cultivés, ils ne trouvent aucune réception. II leur est ainsi impossible de solder l'équation du don sur laquelle est fondée au moins en partie la relation de médiation pédagogique : aucun retour ne vient de la part d'usagers qui évoluent seuls et chez qui la mémorisation (contre-don différé) des chemins de recherche est pratiquement inexistante. Encouragés par cette situation, les bibliothécaires ont tendance à s'enfermer dans une logique techniciste où seul compte l'outil ou bien à privilégier une approche par la conservation des ressources plutôt que par leur circulation et diffusion.

A l'inverse, les usagers fidèles aux postes informatiques ne mettent pas en œuvre des formes d'appropriation techniques ou savantes : ils ne sont réceptifs qu'à ce qu'ils connaissent déjà. Par exemple, la plupart des visiteurs 
Formes de réception et d'appropriation des ressources numériques en milieu étudiant

considèrent qu'ils ne sont pas concernés par les affichages en place sur la formation à la recherche informatisée, les possibilités proposées pour se familiariser avec l'environnement numérique. En cela, ils se distinguent des jeunes chercheurs qui, plus avancés dans le cursus (Master 2 et doctorat) sollicitent l'aide des professionnels, mais à distance et de manière ponctuelle ${ }^{1}$. Sur le versant de la recherche documentaire, c'est moins le lieu qui compte que son environnement et les interfaces: les chercheurs avancés interrogent à distance le catalogue et les banques de données et lisent désormais de manière cloisonnée, hypersélective et ciblée, sans se déplacer. Ils ne semblent pas pour autant sensibles aux sollicitations à la formation en méthodologie de recherche documentaire: ils réagissent en fonction d'une demande particulière et ne prennent pas la documentation assez au sérieux pour songer à la nécessité d'une formation.

\subsection{La réception des ressources numériques : nouvelles pratiques, nouveaux " chercheurs »}

Un récent rapport publié en janvier 2008 par l'University College of London sur les comportements des futurs chercheurs (ceux de la "génération Google ») insiste sur les nouvelles pratiques adoptées par les jeunes usagers des bibliothèques virtuelles pour la recherche et la lecture en ligne (CIBER, 2008). Les traits saillants de ces pratiques tiennent en deux mots : approche extensive des sources et lecture en diagonale des documents. Les chercheurs de la génération Google font des recherches, consultent et lisent de manière très différente des chercheurs de la génération papier: l'immédiateté et la facilité d'accès aux documents semblent les avoir socialisés à un rapport plus direct au texte et, surtout, à une relation plus spontanée au savoir. La recherche par des moteurs généralistes (Google ou Yahoo), la lecture des titres et des mots-clés, l'importation d'abstracts se confond avec la production d'écrits miréférencés, mi-personnalisés, où l'évaluation des sources n'est pas un critère primordial. Dans un rapport récent de I'Online Computer Library Center (OCLC, 1996), on apprend que $89 \%$ des étudiants de premier cycle débutent une recherche documentaire en utilisant un moteur de recherche $(2 \%$ seulement passent d'abord par le site portail d'une bibliothèque) et que $93 \%$ sont satisfaits de cette pratique. Le jeune chercheur peut être simultanément un internaute quelconque et un producteur intellectuel, ce qui rend sans doute moins lourde la démarche scientifique.

\footnotetext{
${ }^{1}$ Comptes nomades, renseignement et aide à la recherche documentaire électronique Sés@me, blog de recherche documentaire http://scdmirailrechdoc.wordpress.com
} 
Mariangela ROSELLI

Quelques données chiffrées recueillies par le Service Commun de la Documentation de l'Université de Toulouse-Le Mirail donnent une idée de la réception des ressources en ligne et laissent entrevoir la manière dont elles sont utilisées. Archipel, le catalogue informatisé du réseau des BU de Toulouse, a été consulté 1927295 fois en 2007, intra muros et depuis l'extérieur ; il s'agit d'un outil qui est interrogé massivement et régulièrement. Les 74 bases de données électroniques ont été consultées 44660 fois, avec 173981 requêtes et 664951 unités téléchargées. Le nombre d'articles téléchargés est de 59406 , avec un engouement prononcé pour la presse d'actualité (Europresse) et moindre pour les articles scientifiques (Academic Search Premier, PsycArticles). Les données relatives au type d'usager de ressources en ligne sont moins bien renseignées que les données disponibles pour les lecteurs d'imprimés. On ne sait pas, par exemple, si les interrogations relèvent de la pratique intense et récurrente des mêmes usagers ou d'un public très étendu, vu qu'aucun indicateur n'est utilisé pour qualifier l'auteur de la consultation ou la requête. Trois indicateurs toutefois permettraient facilement de renseigner la nature des pratiques de consultation s'ils étaient enregistrés et dûment étudiés. Le premier est la formulation du besoin en information qui se traduit par les stratégies de recherche mises en œuvre à partir de mots indexés. Une analyse des mots rentrés en début de recherche (recueillis sous forme de «brouillons, tentatives et échecs de recherche " dans la grille d'observation élaborée pour notre enquête) montre que les jeunes internautes ne savent pas formuler leurs interrogations, ni établir une stratégie de recherche. Ils se contentent bien souvent d'interroger les moteurs avec des mots du langage courant et, à la première difficulté, abandonnent ou changent de chemin. Rares sont ceux qui s'adressent à un bibliothécaire et ceux qui le font ne se donnent pas toujours le temps de s'intéresser à la démarche au-delà de la réponse ponctuelle. L'idée répandue est que les compétences nécessaires pour faire une bonne recherche documentaire sont aussi difficiles à acquérir que le travail de production scientifique lui-même. Conséquence : on bricole soi-même ou on se fait aider au cas par cas. Le deuxième indicateur consisterait à garder une trace des bifurcations, réorientations et arborescences suivies par les internautes de manière à pouvoir analyser de manière systématique et comparative dans le temps leur représentation de l'organisation de l'environnement numérique. Ce paramètre, nommé " parcours dans l'arborescence des ressources » dans notre grille d'observation, a été suivi chez les usagers que nous avions sélectionnés : il donne à voir une représentation simpliste de la cartographie des ressources en ligne, notamment des catalogues fédérés, qui se répercute sur les pratiques récurrentes de recommencement, réorientation, bifurcation sur un chemin qui éloigne de la demande initiale et a plutôt tendance à égarer le jeune chercheur. Le troisième indicateur, lui aussi facile à mettre en place en termes de nombre 
Formes de réception et d'appropriation des ressources numériques en milieu étudiant

et de niveaux d'écran interrogés, concerne les logs et la manière dont ils sont lus. Les observations montrent que les usagers ne lisent pas l'information trouvée, mais préfèrent cliquer sur tous les liens présents sur la page. Cette tendance est particulièrement marquée chez les garçons qui, transférant dans la recherche documentaire, leur préférence pour la lecture zapping et sélective, développent un rapport genré aux informations et aux images sur écran, caractérisé par son immédiateté et sa volatilité (Hersent, 2003).

Bien que les résultats issus de notre enquête de terrain ne soient pas généralisables, on peut avancer un certain nombre d'hypothèses compte tenu de ce que d'autres auteurs ont trouvé (de Singly, 2004 ; Lardellier, 2007). Concernant le rapport à la lecture de textes scientifiques, on constate $1 /$ une recherche extensive et pluridirectionnelle où l'évaluation des sources n'est pas un critère déterminant, $2 /$ une lecture sélective et relativement bridée, au sens où seuls des mots-clés déjà connus fonctionnent comme critère de choix. D'où une importance accrue de pages téléchargées, interfaces communicationnelles et promotionnelles, à faible contenu scientifique. Observations à l'appui, beaucoup plus de temps est passé à chercher et à consulter les pages de présentation, les sommaires, les parcours à renvois qui ne proposent pas de textes, qu'à la lecture effective d'un document, bien que la conscience de ce temps soit très faible (Pedler et Zerbib, 2001). Concernant le rapport à l'environnement numérisé, la comparaison offre/utilisation indique, en premier lieu, une démarche généraliste dans la recherche des sources; en deuxième lieu, des cheminements qui ne s'appuient pas sur le critère de l'exhaustivité des bases de données mises à disposition ; en troisième lieu, une méconnaissance persistante de la méthodologie de recherche en ligne et, en quatrième lieu, un impact très faible de l'environnement numérisé et en cours de numérisation sur les pratiques des jeunes chercheurs, même lorsqu'ils sont avancés. Ne trouvant pas les catalogues des bibliothèques d'une utilisation intuitive, ni les sites portails interrogeables selon des logiques transférables de classement des objets scientifiques (lexicale, thématique), ils préfèrent utiliser des outils familiers du type Yahoo ou Google.

Tout se passe comme si la production intellectuelle se scindait désormais en deux étapes séparées, l'une, ouverte et peu exigeante, de recherche et d'importation de savoir en libre circulation ; l'autre, plus fermée et centrée sur la communauté scientifique (équipe de recherche, spécialistes et experts), d'intégration d'un habitus spécialisé. Celui-ci est moins le résultat de lectures croisées débouchant sur un raisonnement dialectique que de la participation à des réseaux spécialisés qui garantissent une affiliation et une reconnaissance. Ce sont les modalités d'initiation au travail de recherche et la nature même de cette activité qui ont modifié les pratiques des chercheurs. L'offre électronique 
Mariangela ROSELLI

des ressources semble avoir simplement accéléré le processus de spécialisation et de communautarisation des pratiques et des carrières liées à la recherche scientifique. D'un point de vue matériel, ce mouvement se traduit par un retrait progressif du jeune chercheur des espaces ordinaires des relations professionnelles et sociales (campus : enseignement, bibliothèque, mais aussi espace citoyen : affiliations sociales et politiques, associatives et syndicales) au profit d'interstices plus réduits mais beaucoup plus stratégiques. La fréquentation des réseaux sociaux et scientifiques est facilitée et démultipliée par la dimension réticulaire et virtuelle qu'offre la toile, outil direct, rapide et efficace. Pourquoi ces jeunes chercheurs perdraient-ils leur temps à se déplacer en $\mathrm{BU}$ alors qu'ils disposent de moyens plus directs (collègues, équipes de recherche, connaissance du champ spécialisé et des auteurs qui comptent) et plus sélectifs ? Pour ce public, à la fois avancé en production scientifique et balbutiant en méthodologie de recherche documentaire, une sensibilisation ad hoc (ciblée, ponctuelle et sur un objet de recherche précis) est la seule action envisageable pour passer d'une inconscience heureuse à une posture plus réflexive.

\section{Numérique et raccourcis : variations autour de l'abondance de ressources}

Alors que bon nombre de consultations de ressources en ligne (tout particulièrement les bases de données) restent de simples et souvent uniques tentatives, tout se passe comme si l'aveu de l'échec ou de l'abandon était impossible et indigne dans une bibliothèque universitaire et que la " compétence " dans la recherche documentaire devait être à tout prix sauvegardée, et ceci quel que soit le statut de la personne interrogée (étudiant en licence, master ou en doctorat). Une véritable philosophie du " bricolage " tend à s'imposer comme norme rhétorique (valeur défendue) dans les entretiens et comme forme de rationalité (pratique) dans le travail de recherche des sources. II n'en reste pas moins que tous les étudiants qui naviguent sur le Web en $\mathrm{BU}$ font des tentatives répétées pour tracer des chemins vers des documents pertinents, en cohérence avec un but qui est le plus souvent lié à des objets d'étude. Tout au long de notre enquête, nous avons adopté une posture attentive à ces usages en apparence relâchés et sans objectif. Nous pouvons affirmer avoir toujours été en présence d'une préoccupation de cohérence avec une intention, de pertinence par rapport à un thème de recherche, même si les modalités de gestion des difficultés que le Web présente sont résolues de manière très diverse.

A partir de deux portraits, nous esquissons deux profils-types d'usagers de l'environnement numérique de la BU. Un portrait d'étudiante en Master 2

tic\&société - 4 (1), 2010 
incarne l'usager du quantitatif heureux, organisé selon une rationalité de la synthèse juste et efficace. L'autre portrait montre un cas moins heureux, celui d'un étudiant qui redouble l'année de Master 1: livré à lui-même dans un système où le seul levier de réussite est l'autonomisation et la responsabilisation individuelle, il fait du sur place, malgré la pratique intense et ancienne de recherche informatisée. Les deux étudiants ne sont pas comptés parmi les lecteurs de la BU puisqu'ils n'activent pas leur carte ne laissant aucune trace de leur passage sauf leur présence régulière et leur travail sur écran. La première étudiante ne travaille qu'en box, derrière une barrière de chaises, et reste en BU juste le temps d'effectuer ses recherches. Le deuxième étudiant, en revanche, change souvent de place passant d'une station debout à l'entrée de la BU (messagerie personnelle) à une station assise prolongée sur un poste avec accès Internet, avant de s'installer au $3^{\text {ème }}$ étage, le plus souvent à proximité du bibliothécaire : "parce que ça [l']aide à [se] mettre au travail ", selon ses propres termes.

\subsection{Portrait de Camille : "Moi qui n'ai jamais pu lire un livre en entier 》}

Camille, 25 ans, étudiante en géographie, Master 2 Professionnel « Ville et territoires ", rencontrée lors d'une consultation informatique dans un box à côté de la salle des thèses, est une fidèle du poste. Etudiante modèle (assiduité aux cours, travail sérieux, résultats sans surprise, parcours sans accroc), Camille a pourtant un rapport aux textes minimaliste et un rapport à la lecture très difficile. Incapable depuis l'adolescence de terminer un seul livre, elle a érigé ce handicap en principe de survie : face à la nécessité scolaire et universitaire de puiser dans des sources écrites en grand nombre, elle s'est spécialisée dans la recherche de formats de synthèses. La recherche documentaire est, pour Camille, autant une quête d'articles et ouvrages déjà traités par les professionnels des bases de données qu'une avancée toujours plus poussée vers la compréhension des principes d'organisation de ces données: elle a développé une culture des chemins qui mènent, par un simple clic, d'une catégorie générale aux cas particuliers, d'un sous-domaine scientifique à un autre dans une logique parfois hiérarchique, parfois transversale. L'étudiante a développé une compétence dans la recherche documentaire autant qu'une capacité critique vis-à-vis des procédures de recherche.

Avec un usage de la BU très organisé, Camille a un comportement d'usager volatil mais expert : elle consulte, mais elle n'emprunte jamais (elle ne ressent pas le besoin d'avoir une carte de lecteur, elle ne fait donc pas partie des inscrits de la bibliothèque). Pourtant elle a, mieux que tout autre usager, bien saisi l'avantage des ressources documentaires numériques dont elle allie la 
Mariangela ROSELLI

rapidité, l'accès direct des contenus, la forme synoptique des pages de présentation et la synthèse qu'offre le format " abstract ». Les outils numériques correspondent parfaitement aux habitudes de travail de Camille qui s'y est glissée comme dans une seconde peau : elle ne parcourt qu'une partie infime des documents consultés ; elle passe autant de temps à leur recherche qu'à la consultation effective du document; elle "scanne" le titre, le résumé et les informations visuellement mises en relief des périodiques en ligne dont elle ne fait jamais une lecture longue. C'est une autre façon de lire, qui accorde plus d'importance aux mots de la recherche dans le corps du document qu'au contenu, plus aux images et effets visuels (graphiques, schémas, surlignages) qu'au texte linéaire. La linéarité de la page imprimée qui représentait un écueil dans la lecture des ouvrages pour Camille a été écartée pour un autre mode de lecture, à la fois diagonale et analytique. En cela, les indices graphiques fonctionnent comme les signes d'une autre rhétorique du texte qui n'a plus rien à voir avec les conventions de l'imprimé (table des matières, notes de bas de page, références internes au texte). Notons « l'oubli » des guillemets dans cette manière de cheminer vers la constitution d'un document avec signature personnelle.

Les textes d'origine ne sont ici ni une référence d'autorité ni une source incontournable. Ce sont les apports du débat scientifique qui rendent stratégique la recherche documentaire informatisée. L'usage des guillemets est oublié parce que la page à l'écran désacralise le rapport de propriété auteur/texte et qu'elle universalise en quelque sorte la posture de lecteurscripteur. Comme dans l'antiquité où l'écriture philosophique consistait à reprendre les avancées des auteurs avant de présenter un point de vue personnel, nous sommes ici en présence d'un vaste corpus qui se prête à l'emploi dialectique. Le cheminement de Camille consiste à explorer les pistes en ayant bien en tête le résultat à atteindre: il s'agit d'un cheminement dialogique entre les textes, où les signes d'emprunt sont devenus superflus. Elle entre dans le dialogue entre auteurs en écrivant elle-même "à la manière " des textes scientifiques (renvois, références, discussion critique des concepts et utilisation rationalisée des mots-clés). D'ailleurs, la page Web est attentivement étudiée et utilisée à partir des indices proposés (menu, présentation hiérarchique des pages, onglets, scrolling et liens hypertextes), métaphores graphiques qui permettent à l'étudiante de comprendre l'organisation des données et de les explorer en tenant compte de cette organisation proposée en amont par les concepteurs des sites et des bases de données. Ce que l'on observe en la regardant faire est une activité de détection-importation-réécriture plutôt qu'une lecture au sens traditionnel du terme. Les possibilités offertes par le Web, « incarnées » par l'écran, ont comme libéré la créativité intellectuelle de 
Formes de réception et d'appropriation des ressources numériques en milieu étudiant

Camille qui, lectrice complexée, se retrouve étudiante brillante, encouragée par les enseignants dans son activité de revue de littérature scientifique.

De résumés en synthèses, cette étudiante a appris à se passer complètement des lectures intensives et approfondies et développé une spécialisation dans la recherche de pages de synthèse. Pour les articles en ligne, elle montre comment elle procède : étude détaillée de la bibliographie et repérage des autres articles de revues spécialisées (références de sites, références d'autres articles en ligne). Si cette étape est franchie avec succès (c'est-à-dire : si les références d'articles sont assez récentes et réparties sur plusieurs titres de revue), l'article est imprimé et Camille s'installe pour lecture sur une des tables à côté du box. Débute alors la lecture détaillée de la bibliographie avec prise de note (à la main et sur un carnet spécial " références ») classées par thèmes des auteurs, des titres et dates, mais surtout des mots-clés contenus dans les articles tels qu'ils sont résumés, restitués, discutés par l'auteur. Si la revue de littérature semble suffisamment dialectique (présentation contrastée des positions des auteurs, inscription de ces auteurs dans des écoles de pensée, discussion critique des concepts ou des méthodes), la dernière phase est envisagée : la recherche réticulaire des références, des auteurs de sorte à délimiter un espace virtuel de débat intellectuel auquel Camille a l'impression de participer puisqu'elle sélectionne les entrées, les directions, les bifurcations à prendre.

Tout se passe comme si l'interface que permet le Web entre les documents proposés et le dossier "Mes documents " (où Camille importe et travaille ses propres documents) se doublait d'une activité de sélection et de recomposition des textes en vue d'un écrit personnel conforme aux normes d'écriture scientifique. En effet, à un certain moment, la lecture croisée et extensive des abstracts, bascule vers une lecture plus intensive, un approfondissement qui s'inscrit dans un enjeu épistémologique. L'activité de l'étudiante pourrait difficilement être résumée en un copier/coller ou un collage intelligent. Nous sommes en présence d'une lecture analytique doublée d'une réécriture autour d'un objet d'étude qu'elle cerne avec précision. Non seulement toute l'activité sur le Web est fondée sur une réflexion préalable autour de l'objet d'étude, mais elle reflète aussi un travail en amont sur le cheminement à suivre sur le Web pour trouver les ressources adaptées. La différence avec l'écrit traditionnel est que l'écrit de Camille est raisonnablement composite (supports et sources contrôlés et validés), calé sur des débats et des concepts qui lui permettent de se situer rapidement au cœur des enjeux théoriques et, spécificité déterminante, immédiatement élaboré selon les normes de l'écriture scientifique.

Nous sommes ici en présence d'une pragmatique du raccourci et de la synthèse que cette étudiante a transférée de certaines des collections 
Mariangela ROSELLI

imprimées qu'elle privilégiait (thèses, Que sais-je?) aux ressources numériques. Animée par une sorte de culpabilité envers ce qu'elle considère comme l'éthique de lecture d'une bonne étudiante (qu'elle voudrait incarner sans tricher), elle compense son incapacité à lire par le nombre et la qualité des références (articles de revues scientifiques, références récentes, références à des sites spécialisés). Contrairement à d'autres étudiants, la représentation de la cartographie des ressources en ligne chez Camille n'est ni simpliste ni intuitive : elle sait que les interrogations ne peuvent pas être formulées avec des mots du langage courant et que le passage par les moteurs de recherche généraliste l'oblige à faire des détours. Les traits saillants du cheminement que Camille trace dans la recherche sur le Web peuvent se résumer en trois points: une représentation complexe de la cartographie des ressources en ligne, avec une exigence de compréhension des logiques d'organisation implicites ; une reformulation nécessaire des questions initiales en fonction des principes de classification des thésaurus et des bases de données, en dépassant l'écueil d'une définition restreinte d'un objet ou d'un problème; une lecture synoptique des documents et un rendu synthétique mais personnel. Ces trois opérations participent d'un processus d'appropriation du savoir qui est réitéré et testé régulièrement. II finit par fonctionner comme une technique de recherche fondée sur la détection de documents et leur réécriture. L'intérêt même de cette stratégie est de rendre la lecture pleine superflue et de rétrécir considérablement le temps passé sur les textes: l'urgence avec laquelle Camille aborde et sélectionne les textes conduit non pas à une attitude d'interprétation nuancée des contenus, mais à une qualification et à une hiérarchisation des textes. II s'agit d'une posture que d'autres auteurs ont qualifiée de "critique » et elle s'inscrit dans un projet méta-éditorial du texte (Souchier et al., 2003). C'est en ce sens que cette étudiante présente tous les traits caractéristiques des experts en BU : volatile, critique vis-à-vis de ses manipulations et exigeante par rapport à son objectif final.

Le cas de Camille n'est pas unique ; nous avons rencontré des doctorants et des enseignants-chercheurs qui ont un rapport à la lecture aussi négatif et angoissé, mais qui ont, eux aussi, déployé des stratégies d'évitement des textes (longs) ou de contournement de la littérature (par les colloques) ou encore de sélection en amont des sollicitations à lire (mise en place de veilles documentaires très verrouillées). L'acte de lecture n'est plus isolé, spécifique, chronologiquement antérieur à l'écriture; noyé dans un faisceau d'autres activités, elles-mêmes mixtes et innovantes, le moment de la lecture perd en tension, est neutralisé parce que dilué parmi d'autres activités, est de fait moins statique. Le soi n'est plus nécessairement isolé, ce qui contribue à soulager l'anxiété de la solitude intellectuelle. On n'est plus seulement dans la réception, 
on peut simultanément inventer par soi-même, désamorçant l'angoisse générée par le retour au statut d'apprenant.

Certains de ces étudiants sont même devenus des lecteurs à l'écran, faute de pouvoir se mettre à l'imprimé, et développé de réelles compétences à s'orienter dans l'espace ouvert du Web et imprimer une marque subjective forte dans la direction que suit la navigation en vue d'une recherche ciblée. En s'appuyant sur ces compétences, ils copient, coupent, croisent, rebondissent, déjouant ainsi l'angoisse face à la page et à la posture de lecteur : ils écrivent, discutent, créent en même temps qu'ils lisent, le texte ainsi produit présentant non seulement une cohérence mais une implication personnelle. Leurs écrits sont brillants parce que stratégiquement construits selon des formats à fort degré de rationalisation et de schématisation et sur des contenus courts qui posent clairement les enjeux du débat scientifique ou épistémologique dans lequel ils se situent. L'avantage de l'interface que représente le document actif trouvé sur le Web est de permettre à l'usager d'importer, en même temps que les contenus, les formats et les normes d'écriture propres à certains styles de production : ce qu'a parfaitement compris Camille qui, en bonne étudiante en Master 2 Professionnel, écrit comme une stagiaire professionnelle en aménagement urbain, mais ne dédaigne pas les renvois à des débats théoriques. Elle a trouvé un style d'écriture, à mi-chemin entre la rhétorique conventionnelle des textes en sciences humaines appliquées et le style télégraphique qui lui est personnel. L'activité de recherche sur le Web lui a non seulement permis de lire des textes, mais lui a ouvert une voie que l'imprimé n'aurait pu lui offrir, l'écriture synthétique.

\subsection{Portrait d'Aziz ou l'internaute abandonniste}

Aziz, 26 ans, $2^{\text {ème }}$ année de Master 1 de Sciences de l'éducation, fort utilisateur d'Internet en BU où il arrive avec son portable. Septième d'une fratrie de huit enfants, il a été socialisé très tôt aux bibliothèques publiques dont il n'utilise toutefois que les supports les plus visibles et les plus immédiats (Internet et dictionnaires).

Ayant intériorisé la distinction bon usage/mauvais usage du lieu et des ressources, Aziz se place immédiatement du côté des " mauvais " usagers, de ceux qui détournent la vocation première de la BU qu'il associe spontanément à la lecture savante. Aziz insiste particulièrement sur l'apprentissage pénible de l'autonomie, hors de tout soutien, processus dans lequel il se débat toujours et où la seule règle est la survie par la débrouille. Socialisé aux études de manière précoce et quasiment en autodidacte, Aziz fait partie des nombreux étudiants qui, peu encadrés en début de cursus universitaire, ne réagissent pas assez 
Mariangela ROSELLI

rapidement et de manière stratégique pour s'adapter au système des enseignements universitaires. II a, par exemple, intériorisé une habitude qui ne prend pas en compte les degrés de compétence ou les paliers de réussite. Par exemple, il refuse de s'évaluer en termes d'avancement dans le cursus ou de niveaux d'études: l'idée même de progression obligée, d'accumulation de savoir et d'abstraction dans le raisonnement le fait réagir avec véhémence, montrant bien à quel point la représentation lettrée du savoir peut faire violence dans certains cas. Or beaucoup de bibliothécaires, lorsqu'ils se trouvent en position d'apporter de l'aide, considèrent que la bibliothèque est le lieu par excellence pour se glisser dans la peau du chercheur.

Dans les propos des bibliothécaires, la "recherche », au sens noble du terme, désigne d'abord la démarche de collecte de documents et d'informations sur un sujet précis, exercice proposé le plus souvent par un enseignant. Cette démarche est censée alimenter une réflexion personnelle qui encourage l'élève devenu étudiant à élaborer des réponses aux questions posées, sans croire que les solutions se trouvent toutes faites, toutes prêtes, n'attendant que d'être cueillies dans les ouvrages consultés. Dans cette vision, l'idée même de recherche renvoie à une aptitude et à un besoin, inscrits dans l'essence du sujet, d'orienter de manière autonome son intérêt vers le monde et vers soi. Pourtant cette posture, sans être forcément ignorée, n'est pas souhaitée : comme Aziz, la plupart des étudiants que nous avons rencontrés se défendent contre les tentations de la recherche sous sa forme réflexive (ouvrant sur la béance d'une quête métaphysique), se détournent des ateliers de formation à la recherche (l'élisant à une méthode) et se méfient de l'injonction à suivre un passage obligé pour avancer dans le monde du savoir et de la réflexion. Chez Aziz, par exemple, les étapes sont exploratoires, les avancées discontinues, inégales dans le temps et inégalement utilisables pour ses études, fonctionnant en boucle et non à sens unique.

Tâtonnements, retours et abondons

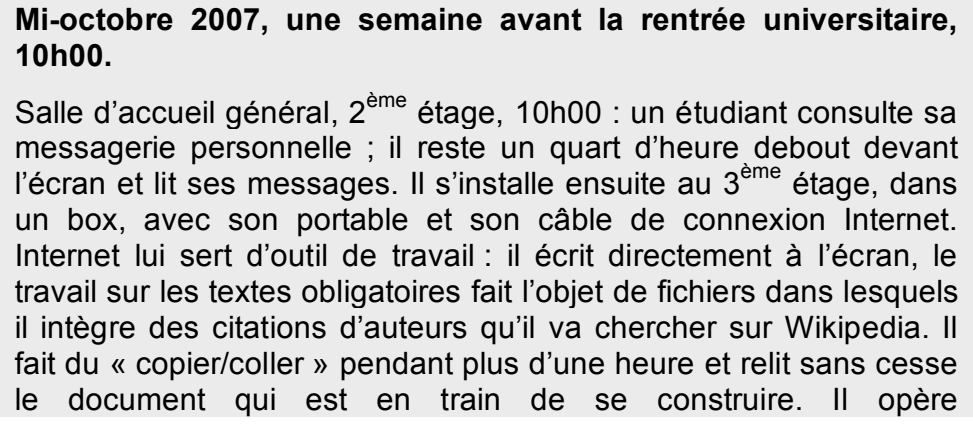
un box, avec son portable et son câble de connexion Internet. Internet lui sert d'outil de travail : il écrit directement à l'écran, le travail sur les textes obligatoires fait l'objet de fichiers dans lesquels il intègre des citations d'auteurs qu'il va chercher sur Wikipedia. II fait du " copier/coller » pendant plus d'une heure et relit sans cesse le document qui est en train de se construire. II opère 
Formes de réception et d'appropriation des ressources numériques en milieu étudiant

simultanément une lecture des documents trouvés et une mise en cohérence de son propre texte. Après une longue pause-cigarette sur la passerelle d'entrée pendant laquelle il discute avec une fille, il revient et change d'activité : il fait d'abord des recherches bibliographique à partir de Google Scholar, puis il revient sur Archipel. II trouve des notices bibliographiques qu'il réutilise pour cerner un ensemble de mots-clés, mais ne lit pas les textes. Puis il revient à Google et part sur des sites institutionnels (Ministère de la culture, Musées de France) qu'il visite longuement, en poussant jusqu'au $3^{\text {ème }}$ niveau d'écran. II revient systématiquement aux pages d'accueil pour vérifier qu'il a bien cliqué sur tous les renvois. L'impression est d'une consultation approfondie, mais il nous dira qu'il n'a pas trouvé exactement ce qu'il voulait. Sur le site des Musées de France, il clique trois fois sur un onglet, se perd en chemin, n'aboutit jamais au même point, reste un peu plus de dix minutes sur le dernier point d'arrivée (une image introduisant le site d'un musée parisien), ferme tout et abandonne avec un air dépité, la photo du site lui ayant servi de critère de " pertinence négative " (Boubée, 2007). Regarde la montre, descend aux toilettes, revient, recommence en partant d'un autre site. II passe plus de deux heures à consulter de manière erratique des cheminements aléatoires, qu'il découvre au fur et à mesure. Au cours de l'entretien qui suit, il affirme n'être resté qu'une demi-heure devant l'écran.

Comme s'il n'avait pas vu le temps passer, Aziz incarne la figure de l'internaute amateur en recherche documentaire. Par sa relation au temps et ses stratégies de quête sur le Web, il adopte ce que d'autres ont appelé " une posture capitalisante » avec un projet pragmatique (Souchier et al., 2003) : souci du quantitatif pour atteindre un résultat correspondant à une représentation préalable des éléments nécessaires à une information complète. II cherche beaucoup et longtemps, deux critères qui lui permettent de dire qu'il fait de la recherche. Seulement, il ne trouve pas toujours ce qu'il cherche et il abandonne au bout de "quelque temps ", quand il se rend compte qu'il n'est pas sur la bonne voie. Son parcours de lecteur n'est ni chrono-logique ni méthodo-logique, mais composé d'arrêts fréquents ou d'abondons. Mais ceux-ci constituent des moments-clés à eux seuls : les abandons, parce qu'ils poussent Aziz à finir une opération pour mieux la recommencer et l'instabilité de certaines pages Web encouragent dans une certaine mesure cette attitude de curiosité ; les arrêts, parce qu'ils laissent le temps de décoder, de dépouiller les propositions pour mieux comprendre les tenants et les aboutissants d'une question.

Le chemin suivi par Aziz comprend ainsi deux temps. Le premier est un temps d'exploration et de sélection restrictive contre les tentations de dispersion. Le deuxième est un temps d'importation dans un document 
Mariangela ROSELLI

personnel de différentes sources qui mélange le plus souvent les genres et les supports (images et son avec texte). La stabilisation des données passe toujours par l'impression papier avec récapitulatif des sites visités, des sources consultées et des documents importés. Le papier fournit à la fois le résultat des recherches et la preuve du travail accompli.

En autodidacte, Aziz a intériorisé le respect des sources et le rapport fondateur aux textes d'auteur : il achève son activité de recherche informatisée toujours par une liste sélective des sources retenues et par l'enregistrement du cheminement de recherche qui lui a apporté le plus d'éléments utiles (utilisation répétée et appréciée de la flèche de retour en arrière). Le cheminement a pour Aziz valeur pédagogique en soi, et non comme pour Camille, valeur stratégique en termes de contenus. Aziz présente plusieurs traits caractéristiques de l'autodidacte, mais l'intérêt qu'il porte aux aspects cognitifs des opérations de recherche est particulièrement présent chez lui. La manière de construire les savoirs l'intéresse tout autant que les savoirs eux-mêmes. Contrairement aux textes de Camille qui sont conçus pour être rendus, les textes produits par Aziz sont des documents pour soi. Ils servent à digérer les contenus. Le cheminement suivi par Aziz tend à réduire le nombre de ressources mais surtout à ralentir le rythme jugé trop élevé des propositions : il s'agit d'une technique de réduction et de ralentissement de l'apport de savoir proposé par le Web. L'acte de cliquer est d'ailleurs significatif : en agissant sur la machine, le clic donne l'impression d'agir sur les contenus visibles à l'écran et permet d'avancer par àcoups, traduisant les tâtonnements, les incertitudes, les décisions prises in fine.

De la même manière, son parcours d'étudiant se déroule par à-coups (ce que le système appelle " échec » dans les statistiques des examens) ; composé de retours et détours sur un cursus (tout comme sur un ouvrage lu, relu plusieurs fois), selon un mouvement progressif/régressif permanent. Pendant sept années, depuis le baccalauréat, Aziz s'évertue à s'approprier un cursus, celui des sciences de l'éducation, et à donner du corps à son projet (devenir professeur des écoles). Pour donner du sens à cet itinéraire, il a mis en place un certain nombre de tactiques. La première vise à déjouer la peur de clore un savoir pour en aborder un autre inconnu: une rationalité du cloisonnement se charge de compartimenter la vision du savoir et de le rendre plus abordable. La deuxième tactique a consisté à classer tout ce qu'il apprend à sa façon, par thématiques, fichiers, auteurs qu'il rebaptise avec des titres suggestifs. La classification Dewey dont se servent les bibliothécaires pour classer et ranger les ouvrages ne l'intéresse pas (il profite d'ailleurs de l'entretien pour me demander ce que peuvent bien signifier les cotes des collections dans les rayons). L'organisation du savoir (en BU tout comme sur le Web) ne l'intéresse pas, montrant par là une distance vis-à-vis des chemins habituels de 
Formes de réception et d'appropriation des ressources numériques en milieu étudiant

construction et mise en ordre des connaissances. L'appropriation demeure chez Aziz une étape qui lui demande beaucoup de temps et beaucoup d'énergies. Afin de faire face à des consignes de recherche et de lecture nombreuses, il a donc résolu de se constituer lui-même un corpus réduit qu'il parvient à maîtriser à force de retours et de répétitions.

Aucun autre cas mieux que celui d'Aziz ne montre à quel point le savoir-faire des bibliothécaires peut peser dans un accès direct aux moteurs de recherches : à défaut d'interaction sollicitée auprès des bibliothécaires, seule peut être efficace une simplicité accrue des catalogues de la bibliothèque, des possibilités plus linéaires, plus conviviales de liens, de renvois, des menus plus transparents. Le site portail peut jouer véritablement le rôle de balise tout en étant un tremplin vers des connaissances plus larges. La question de la valeur des sources n'intéresse qu'une minorité d'étudiants s'étant formé à la grammaire complexe des ressources en ligne. La plupart du temps, l'offre numérique se heurte à des obstacles techniques et intellectuels dont les étudiants (et non seulement les étudiants de licence) ne s'aperçoivent même pas. Le cas de cet étudiant "abandonniste » est exemplaire des éternels débutants en recherche documentaire qui érigent la logique de passage à passage en principe de travail sans pouvoir réunir les conditions de production personnelle.

Le portrait d'Aziz renvoie au profil-type des étudiants qui ne parviennent pas à convertir leur savoir-faire sur la toile en méthode de recherche. Comme beaucoup d'autres usagers de la BU que nous avons observés aux prises avec des renvois, onglets et (sous-)connexions, il est perdu au bout du $3^{\text {ème }}$ niveau d'écran. Les bifurcations proposées, les arborescences, les sous-classifications tendent des pièges où se perdent la majorité des bricoleurs, sans pouvoir admettre ou avoir conscience des passages ratés et des autres possibilités. Pour ces usagers, les parcours de recherche sont personnels, presque intimes. Dans tous les cas, ils sont liés à des recherches larges, des intérêts ouverts, où se confondent recherche d'information et recherche de documentation. C'est pourquoi les échecs avoués sont rares: ces internautes sont ravis de la plénitude et de la liberté qu'ils perçoivent par rapport à la contrainte du papier, du texte de référence. Dans cette liberté nouvellement acquise, l'individu se voit comme tout-puissant: le sentiment immédiat que l'on peut retirer d'une recherche sur Google n'est presque jamais un sentiment d'échec. Par comparaison avec le livre, contraignant et inerte, synonyme de travail et d'univers scolaire, les nouvelles technologies sont caractérisées par leur ouverture et leur « appel à la curiosité » (rien n'est jamais achevé), leur interface ludique, l'interactivité proposée, la facilité à travailler en groupe lorsqu'on navigue. Une bonne partie des étudiants rencontrés dans le cadre de l'enquête 
Mariangela ROSELLI

sait télécharger, concevoir des sites, créer un blog, retoucher des photos, travailler à partir de logiciels de musique électroacoustique. Tous ont appris ces compétences seuls ou entre amis, mais aucun en cours ou en famille. Cet apprentissage en autodidactes ou en petits groupes de copains est révélateur de la manière dont ces jeunes ont investi les TICE : de manière volontaire, intuitive et sans complexe. Approche qu'ils transfèrent à l'identique dans la recherche de documentation où ils dénoncent toute forme de bridage, de médiation ou encore d'accompagnement. Comment ne pas voir dans ces résistances une manière de ruser avec le pouvoir lié à la production et à la circulation du savoir?

\section{En guise de conclusion}

Alors que la lecture savante, comme modalité de l'appropriation culturelle, se situe dans le respect des formes instituées du savoir, les pratiques numériques ouvrent la porte à des possibilités de personnalisation et d'intervention directe de l'usager dans les contenus. En cela, le cheminement suivi par l'étudiant Aziz donne à voir comment, avec très peu de moyens en formation documentaire initiale, l'usager peut mettre au point des stratégies utiles pour constituer son corpus de documents. L'intérêt du cas d'Aziz réside dans les techniques d'adaptation et de conversion des contenus savants en contenus appropriés à son projet personnel, à savoir ralentir le rythme des apprentissages et rendre les contenus plus "intéressants " au sens de plus vivants, plus en contact avec la réalité, plus proches de ses centres d'intérêt. L'enjeu est bien un enjeu d'appropriation via un processus d'adaptation : Aziz n'est pas moins actif que Camille du point de vue de l'adaptation entre moyens mis en œuvre et projet poursuivi (se maintenir au niveau exigé mais aussi s'inscrire dans un cadre de recherche). Malgré la lenteur et la redondance de son avancement, il entend venir à bout de son parcours et sortir de l'université avec des compétences majorées (en recherche, en réflexion, en écriture) et non seulement avec un diplôme.

Par contraste, le cheminement - plus rapide mais non moins laborieux - de Camille par rapport à son émancipation vis-à-vis de la lecture traditionnelle, apparaît plus efficace et achevé. On voit bien Camille investir ses compétences en recherche documentaire dans un premier emploi dans un cabinet d'étude d'architectes et géographes. Alors que la méthode d'Aziz est difficile à valoriser et à exploiter en dehors de son cadre individuel. Néanmoins, du point de vue des compétences de réception développées grâce à l'environnement numérisé de la $\mathrm{BU}$, tous les deux font preuve d'un travail d'adaptation : si Camille réécrit les contenus en vue d'un document à présenter aux enseignants, Aziz sélectionne et restreint considérablement l'offre en amont en vue d'un document

tic\&société - 4 (1), 2010 
"facile à lire », souvent multimodal et interactif (beaucoup d'images et de liens importés et organisés). La première lit et écrit pour autrui, le deuxième surfe, coupe, colle et importe pour lui, afin de créer les conditions favorables pour apprendre. Sans cela, il serait depuis longtemps écarté du cursus universitaire. II partage ses produits avec un groupe restreint de camarades qui, comme lui, " sont en galère avec les études et ne se formalisent pas ". Écriture académique, respectueuse des normes versus écriture intimiste et relâchée : du point de vue de la réussite universitaire, les évaluations se chargent de classer les deux styles issus de méthodes bien différenciées. Mais du point de vue de l'appropriation des cheminements et des contenus eux-mêmes, les deux manières de faire semblent chacune en adéquation avec un projet.

Les bibliothécaires nous avaient demandés de répondre à la question suivante : que font-ils à la $B U$ s'ils ne lisent pas ? Trois éléments de réponse se détachent du matériau d'enquête. Premièrement, on peut affirmer que les étudiants, des primo-entrants aux doctorants, ne font pas que de l'Internet ludique et que leurs pratiques de recherche informent directement leurs manière de jouer, confirmant ce que disait Passeron sur les jeux distrayants « qui, plus qu'ils ne détournent le visionnement de son but, l'atteignent de manière détournée » $(1984,34)$. Les étudiants lisent et lisent souvent pour leurs études, mais ils le font de manière détournée, longue, en se perdant parfois. Deuxièmement, l'accès facilité aux informations ne fait pas de celles-ci des connaissances: des processus de connaissance et d'interprétation sont engagés simultanément à une socialisation à l'environnement numérique et à une négociation avec l'intention initiale de l'usager. Seule l'articulation réussie de ces opérations permet de transformer certaines sources en ressources pour soi (Aziz) ou pour autrui (Camille). Troisièmement, le jeu des activités sur le Web croise, contrairement à l'illusion de neutralité, les déterminismes socioculturels en renforçant le poids différentiel de la capacité critique vis-à-vis des sources et de la compétence à la lecture multimodale ; d'où la nécessité d'une politique volontariste de formation sous forme de propédeutique à l'environnement numérisé.

Ces trois éléments déterminent des parcours de recherche et des modes de lecture-étude en BU bien distincts. Nous avons ainsi pu établir cinq profils-types d'usager : 1/ les séjourneurs qui utilisent la BU comme une salle d'étude (révisent et écrivent sur des imprimés importés) et qui constituent le groupe le plus nombreux ; 2 / les errants de l'université de masse, 3 / les internautes ; 4/ les usagers faisant preuve de bonne volonté culturelle et scolaire ; $5 /$ les experts en recherche documentaire qui ressemblent à des météores, à la fois à cause de la rapidité de leurs passages et de leur rareté en BU (Roselli et Perrenoud, 2010). 
Mariangela ROSELLI

Aziz fait partie du deuxième type, les errants de l'université de masse, tandis que Camille présente toutes les caractéristiques que nous avons identifiées chez les experts. Ces deux groupes se distinguent sur deux points. Le premier se situe au niveau du contrôle et de la maîtrise de l'individu sur le système, aussi bien universitaire, pédagogique, administratif que documentaire. Les errants, contrairement aux autres profils mais à l'opposé symétrique des experts, sont "pris » par le système et restent captifs de ses logiques. Ils investissent beaucoup d'énergies et de temps pour s'adapter. Dans le domaine des ressources électroniques plus spécialement, la " prise » sur la machine (Ghitalla et al., 2003) marque une différenciation sensible entre degrés d'appropriation : les étudiants bricoleurs restent durablement "pris » par le système complexe du Web. Tout semble indiquer que ces compétences n'évoluent pas de manière progressive ou cumulative, mais plutôt de manière répétitive, mimétique et restrictive (Brodbent et Cara, 2003). Le rapport au temps est l'autre ligne de partage entre les deux types: tandis que les errants vivent le passage à l'université comme une expérience de vie qu'ils tendent à faire durer (par défaut ou par véritable intérêt), les experts instrumentalisent cette étape comme un passage obligé qu'ils essaient d'accélérer. Ce rapport au temps se reflète dans les pratiques mises en œuvre sur le campus : assiduité au cours, multiplication des options, fréquentation de la BU et des autres espaces de rencontre. Les étudiants comme Camille ont un rapport aux études épisodique et stratégique; ils prennent ce qui leur est proposé mais ne s'attardent pas, ne partagent pas, vivent leur vie ailleurs. Ils obtiennent les résultats qu'il faut au moment voulu. Les errants qui résistent après la première année comme Aziz ont, en revanche, un rapport aux études durable et affectif : à cause du temps d'adaptation qui leur est nécessaire pour se mettre au pas avec le système universitaire, ils s'investissent et s'impliquent sur les lieux, ils occupent les espaces en quête de repères, de normes, d'opportunités. Ils utilisent le campus, ses services et ses ressources, comme un cadre de vie d'où ils doivent tirer un sens. N'étant pas, comme les experts, des étudiants brillants, ils ont tendance à occuper les zones périphériques (en BU comme sur le campus et en cours) pour cacher l'indigence dans les savoirs et le besoin constant d'aide et de conseil. Ils déguisent souvent leurs besoins en attitude butée, voire en refus d'interaction. Les ressources numériques offrent la possibilité d'entrer dans une logique de recherche en restant dans l'anonymat. En cela, elles constituent une bonne fenêtre d'observation pour voir à l'œuvre ce type d'étudiants, généralement réticents à se laisser saisir dans les activités de lecture et d'étude, mais tout à fait ouverts à montrer comment ils savent naviguer et s'orienter sur le Web. 
Formes de réception et d'appropriation des ressources numériques en milieu étudiant

\section{Références bibliographiques}

BOUBEE N., 2007, « L'image dans l'activité de recherche d'information des élèves du secondaire », Spirales, $n^{\circ}$ 40, octobre, pp. 141-150.

BRODBENT S. et F. CARA, 2003, « Les nouvelles architectures de l'information ", in Text-e. Le texte à l'heure de l'Internet, Paris, Bpi/Centre Georges Pompidou, pp. 197-213.

CIBER (Center for Information Behaviour and the Evaluation of Research at University College London), 2008, Information Behaviour of the Researcher of the Future : a Ciber Briefing Paper, 11 janvier, www.ucl.uk/slais/research/ciber/downloads/ggexecutive.pdf

CHARTIER R., 1996, Culture écrite et société. L'ordre des livres (XIV-XVIII siècles), Paris, Albin Michel.

de CERTEAU, M., 1980, L'invention du quotidien, tome 1 : Arts de faire, Paris UGE.

de SINGLY F. (dir.), 2004, Enquête du CERLIS sur les lectures étudiantes réalisée dans le cadre de l'enseignement de méthodes (DEUG II, en 2003 et en 2004, Paris-V), Paris, CERLIS.

DESPRÈS-LONNET M., 2007, « Les stratégies de recherche documentaire des étudiants, entre attentes institutionnelles et pratiques réelles », in PAPY F. (dir.), 2007, Usages et pratiques dans les bibliothèques numériques, Paris, Hermès Science Publications, pp. 203-219.

DIRECTION DES BIBLIOTHEQUES DU MINISTERE DE L'EDUCATION, Enquête Statistique générale des bibliothèques universitaires. Enquêtes consultées : 2005, 2006, 2007.

EISENBERG, M., 2008, « The parallel information universe », Library Journal, $1^{\mathrm{er}}$ mai www.libraryjournal.com/toc-archive/2008/2008501.htlm

Enquête sur le public de la bibliothèque de l'Université de Paris 8, 2007, MV2 Conseil bu.univparis8.fr/web/actualites/enquête\%20usagers\%20bilan\%202007.pdf

HERSENT J.-F., 2003, « Les pratiques culturelles adolescentes : France, début du troisième millénaire ", Bulletin des bibliothécaires de France, $\mathrm{n}^{\circ} 3$, pp. 12 21.

GHITALLA, F. et al., 2003, L'outre-lecture. Manipuler, (s')approprier, interpréter le Web, Paris, Bpi/ Centre Georges Pompidou. 
Mariangela ROSELLI

JOUET J., 2000, « Retour critique sur la sociologie des usages », Réseaux, $\mathrm{n}^{\circ}$ 100, pp. 487-521.

LAHARY D., 2008, http://lahary.wordpress.com/2008/04/22/la-bibliothèquevous-en-donne-moins

LAHIRE B., 1995, Tableaux de familles, Paris, Seuil/Gallimard.

LAHIRE B., 2004, Les transferts imparfaits. La culture des individus : dissonances culturelles et distinction de soi, Paris, La Découverte.

LARDELLIER P., 2007, « Pratiques de lectures étudiantes à l'ère d'Internet » in LARDELLIER P. et M. MELOT (dir.), Demain, le livre, Paris, L'Harmattan, 2007, pp. 47-58.

LE MAREC J. et I. BABOU, 2003, « De l'étude des usages à une théorie des " composites » : objets, relations et normes en bibliothèque ", in SOUCHIER E. et al., 2003, Lire, écrire, réécrire. Objets, signes et pratiques des médias informatisés, Paris, Bpi/Centre Georges Pompidou, pp. 235-299.

MARESCA B., 2005, Enquête sur les pratiques documentaires des étudiants, chercheurs et enseignants-chercheurs de Paris VI et Paris VII, Rectorat de Paris, BIUSJ, rapport du CREDOC, $n^{\circ} 238$.

MARESCA B. et al., Les bibliothèques municipales en France après le tournant Internet : attractivité, fréquentation et devenir), Paris, Bpi/ Centre Georges Pompidou.

OCLC (Online Computer Library Center, ex-Ohio College Library Center), 2006, College students' perceptions of the libraries and information resources, report to the OCLC Membership, Dublin (Ohio).

PASSERON J.-C., GRUMBACH M. et al., 1984, L'œil à la page : enquête sur les images et les bibliothèques, Paris, Bpi/Centre Georges Pompidou.

PASSERON J.-C., 1991, « Le polymorphisme culturel de la lecture », in Le raisonnement sociologique. L'espace non-poppérien du raisonnement naturel, Paris, Nathan, pp. 335-345.

PEDLER E. et O. ZERBIB, 2001, Les nouvelles technologies à l'épreuve des bibliothèques, Paris, Bpi/Centre Pompidou, préface de Roger Establet.

PETITE S. et L. LE DOUARIN, 2008, « Le diversité des usages et des étudiants au sein de la Médiathèque Jean Lévy à Lille », Bibliothèque(s), $n^{\circ} 40$, octobre, pp. 66-68.

POISSENOT C. et S. RANJARD, 2005, Usages des bibliothèques. Approche sociologique et méthodologie d'enquête, Villeurbanne, Cahiers de l'ENSSIB.

tic\&société - 4 (1), 2010 
Formes de réception et d'appropriation des ressources numériques en milieu étudiant

ROSELLI M., 2006, « Usagers et usages devant une offre de lecture publique libre : parcours d'acculturation et formes d'appropriation lettrées », Sociétés contemporaines, $\mathrm{n}^{\circ} 64$, décembre, pp. 135-151.

ROSELLI M. et M. PERRENOUD, 2008, Les publics de la BU de l'Université de Toulouse-Le Mirail, Rapport d'enquête au Service Commun de la Documentation de l'Université Toulouse 2-Le Mirail, disponible sur le site du SCD-UTM.

ROSELLI M. et M. PERRENOUD, 2010, Du lecteur à l'usager. Ethnographie d'une bibliothèque universitaire, Toulouse, Presses Universitaire du Mirail.

SOUCHIER E. et al., 2003, Lire, écrire, réécrire. Objets, signes et pratiques des médias informatisés, Paris, Bpi/Centre Georges Pompidou.

TOUITOU C., 2008, « Les nouveaux usages des générations internet : un défi pour les bibliothèques et les bibliothécaires, Bulletin des bibliothécaires de France, t. 53, n 4, pp. 67-70. 